\title{
Stereoselective analysis of methadone and EDDP in laboring women and neonates in plasma and dried blood spots and association with neonatal abstinence syndrome
}

Ingrid F. METZGER, PharmD*1,5, Anna E. THOMAS, MD², Ms. Cindy A. EVRARD, BSN ${ }^{1}$, David R. JONES, $\mathrm{PhD}^{3}$, Ms. Andrea R. MASTERS, MS ${ }^{4}$, David M. HAAS, MD ${ }^{1,3}$, Laura S. HANELINE, MD², Sara K. QUINNEY, PharmD, PhD'1,3

${ }^{1}$ Department of Obstetrics and Gynecology, Indiana University School of Medicine, Indianapolis, Indiana, USA

2Department of Pediatrics, Indiana University School of Medicine, Indianapolis, Indiana, USA

${ }^{3}$ Division of Clinical Pharmacology - Department of Medicine, Indiana University School of Medicine, Indianapolis, Indiana, USA

${ }^{4}$ Clinical Pharmacology Analytical Core - Indiana University Simon Cancer Center, Indiana University School of Medicine, Indianapolis, Indiana, USA

${ }^{5}$ Present address University of Barilia, Brasilia, Brazil

* These individuals contributed equally to this work.

The authors report no conflict of interest.

This study was supported by K23 HD071134-01A1 (NICHD) and UL1TR001108 Indiana Clinical and Translational Sciences Institute (Pediatric Project Development Team Funding). Analytical work was performed by the Clinical Pharmacology Analytical Core laboratory, a core laboratory of the Indiana University Melvin and Bren Simon Cancer Center supported by the National Cancer Institute grant P30 CA082709. The funders

This is the author's manuscript of the article published in final edited form as:

Metzger, I. F., Thomas, A. E., Evrard, C. A., Jones, D. R., Masters, A. R., Haas, D. M., Haneline, L. S., \& Quinney, S. K. (2021). Stereoselective Analysis of Methadone and EDDP in Laboring Women and Neonates in Plasma and Dried Blood Spots and Association with Neonatal Abstinence Syndrome. American Journal of Perinatology, 38(9), 968-975. https://doi.org/10.1055/s-0040-1701505 
had no role in the identification, design, conduct, and reporting on this analysis. The content is solely the responsibility of the authors.

This work has been presented in part at the American Society for Clinical Pharmacology and Therapeutics $118^{\text {th }}$ Annual Meeting, Washington DC, March 15-18, 2017.

Corresponding Author: Sara K. Quinney, PharmD, PhD

950 West Walnut Street, R2 Room 476

Indianapolis, Indiana 46202

Work: (317)274-2796

squinney@iu.edu

Word Count:

Abstract: 177

Main Text: 3309

Short Title: Methadone exposure and NAS severity

Key Words: methadone, neonatal abstinence syndrome, opioid use disorder, pharmacokinetics 


\section{ABSTRACT:}

Objective: This pilot study evaluated the relationship between maternal and neonatal R- and S-methadone and R- and S-2-ethylidene-1,5-dimethyl-3,3-diphenylpyrrolidine (EDDP) exposure and the severity of neonatal abstinence syndrome. The use of dried blood spots (DBS) as an alternative for plasma in assessing methadone and EDDP was also assessed.

Study Design: Women receiving methadone for medication assisted treatment of opioid use disorder during pregnancy were eligible for recruitment. Plasma and dried blood spot samples were collected from mothers during labor, from cord blood, and from newborn during genetic screen. R-/S-methadone and 2-ethylidene-1,5-dimethyl3,3-diphenylpyrrolidine (EDDP) were measured by HPLC-MS/MS. Associations between methadone exposure, neonatal morphine requirements and severity of neonatal abstinence syndrome were examined.

Results: Twenty women and infants completed the study. Maternal methadone dose at delivery was $112 \mathrm{mg} / \mathrm{d}$ (range 60-180 mg/d). Sixteen neonates experienced NAS requiring morphine; 3 also required phenobarbital. Higher cord blood concentrations of $\mathrm{R}$-methadone, $\mathrm{R}$ - and S-EDDP were associated with higher maximum doses of morphine $(p<0.05)$.

Conclusion: Maternal methadone and cord blood concentration at delivery are variable and may be potential markers of neonatal abstinence syndrome. 


\section{INTRODUCTION}

Opioid use has increased more than four-fold within the last decade,${ }^{1}$ leading to the declaration of a national public health emergency. ${ }^{2}$ Misuse of opioids among pregnant women is common. Opioid misuse in pregnancy often leads to neonatal opioid withdrawal syndrome (NOWS) in the neonate. As a number of drugs, including antidepressants and other illicit substances, may contribute to clinical withdrawal in the neonates, we have employed the more broad term neonatal abstinence syndrome (NAS) in this study. NAS has increased nearly fivefold from 2000 to 2012 . Every 25 minutes a baby is born with NAS in the U.S..$^{1,3}$

NAS is a costly morbidity, most commonly caused by maternal opioid dependence during pregnancy. It is a self-limited constellation of signs and symptoms consisting of neurologic excitability, gastrointestinal dysfunction, and autonomic disturbances ${ }^{4}$. As a consequence of increased use of neonatal intensive care units and prolonged hospitalization for the treatment of NAS, average hospital charges for infants with NAS amount to more than five times those for all other infants ${ }^{1,3,5}$.

To reduce the risk of neonatal morbidity and mortality associated with in utero exposure to heroin and other short-acting opioids, the American College of Obstetricians and Gynecologists (ACOG) and the American Society of Addiction Medicine (ASAM) recommend opioid-assisted therapy with methadone or buprenorphine during pregnancy. ${ }^{6}$ While the use of buprenorphine has increased over the past few years, methadone is still widely used for medication assisted therapy. In $2018,25 \%$ of outpatient opioid treatment programs only provided methadone. ${ }^{7}$ Additionally, there are a number of women who are not candidates for buprenorphine 
therapy, who may be treated with methadone. Although methadone treatment programs improve access to prenatal care and decrease some of the morbidities associated with illicit opioid use, a large number of infants exposed to methadone in utero develop NAS shortly after birth and require pharmacological treatment for withdrawal. ${ }^{8}$

Methadone is a synthetic opioid analgesic that has a long half-life of approximately 22 hours in adults. It is available as a racemic mixture of R- and Smethadone and undergoes $\mathrm{N}$-demethylation to its main metabolite, 2-ethylidene-1,5dimethyl-3,3-diphenylpyrrolidine (EDDP), which is inactive. R-methadone accounts for the majority of opioid effects ( $\mu$-opioid receptors) and has more potent analgesic effect than S-methadone ${ }^{9,10}$. Numerous studies have attempted to link the severity of NAS to maternal methadone dose, but the results have been inconsistent. In a meta-analysis, 19 studies found a correlation between maternal methadone dose and NAS severity, while 18 did not; when limited to those studies that used an objective scoring system to evaluate NAS, no association was found. ${ }^{11}$ Interindividual variability in pharmacokinetics of methadone may lead to the discrepancy between methadone dose and NAS severity. Studies have reported up to 17 -fold difference in plasma methadone concentration among individuals receiving the same dose. ${ }^{12}$ This variability is explained, in part, by pharmacogenomic variants in drug metabolizing enzymes and transporters. ${ }^{13,14}$ Metabolic changes associated with pregnancy may also contribute to variability in exposure to methadone..$^{15-17}$ Cord blood concentrations of methadone are lower than maternal concentrations due to the protective effects of the p-glycoprotein transporter in placenta. ${ }^{18-21}$ There have been a few small studies examining the 
relationship between cord blood methadone concentrations and the severity of NAS which suggest that lower cord blood concentrations and a faster rate of decline in neonatal plasma concentrations correlate to more severe withdrawal. ${ }^{22,23}$ Similarly, in adults a more rapid decline in plasma methadone concentration correlates with increased withdrawal symptoms. ${ }^{24}$ However, data examining the relationship between maternal and neonatal pharmacokinetics of methadone to the severity of NAS are lacking.

Current treatment paradigms for NAS base the need for pharmacotherapy on clinical assessment of the infant, e.g. by using the modified Finnegan score. Earlier identification of infants likely to experience severe NAS may allow for adjustment in clinical treatment algorithms for withdrawal, for example by reducing the threshold modified Finnegan score for initiating treatment, allowing the infant to begin treatment earlier. We hypothesize that maternal and neonatal concentration of methadone enantiomers at delivery may correlate with severity of NAS. Therefore, we undertook a pilot study to evaluate the relationship between maternal and fetal concentrations of individual enantiomers of methadone and its primary metabolite, EDDP, and the risk of neonatal NAS. In addition, we evaluated the use of dried blood spots (DBS) as an alternative to plasma for determining concentrations of methadone and EDDP.

\section{MATERIALS AND METHODS}

Women $\geq 18$ years of age with a singleton pregnancy who had been taking methadone beginning prior to the 28th week of gestation and who planned to deliver at Eskenazi or IU Health Methodist Hospitals were eligible for recruitment into this study. Women were excluded if they had a urine toxicology screen that was positive for other 
opioids at the time of enrollment, liver or kidney dysfunction, or known fetal anomalies or genetic disorders. Enrollment occurred during an outpatient obstetric clinic visit, inpatient hospital stay, or upon admission to labor and delivery. The study was approved by the Indiana University Purdue University Indianapolis Institutional Review Board and written informed consent was obtained from each woman for herself and her infant.

Two blood samples were collected from each subject. Maternal samples were obtained upon admission to the labor and delivery unit for planned delivery and within 1 hour after birth. Infant samples were obtained from cord blood at the time of birth and in conjunction with the newborn screen. Maternal and cord whole blood samples were collected into EDTA tubes and aliquots transferred onto DBS cards (Whatman DMPK903). The remaining blood was centrifuged to plasma and stored at $-80^{\circ} \mathrm{C}$ until analysis. The infant samples obtained during the newborn screen via heelstick were collected directly onto DBS cards. The DBS cards were allowed to dry completely and then placed into a sealed plastic bag with a desiccant packet and stored at room temperature until analysis.

\section{Demographic and clinical data}

Demographic and clinical data were obtained from the medical record. The modified Finnegan score ${ }^{25}$ was used as standard of care to monitor withdrawal symptoms of infants. NAS was determined to be severe and warrant pharmacologic treatment when an infant had three consecutive Finnegan scores $>8$ or one score $\geq 12$. Treatment for NAS at both hospitals followed the same standard protocol which uses oral morphine as the primary medication, with the addition of phenobarbital as 
adjunctive therapy for refractory symptoms. The recommended morphine starting dose was $0.04 \mathrm{mg} / \mathrm{kg} /$ dose every 4 hours. Morphine doses were escalated if needed and weaned according to physician discretion.

\section{Analytical method}

R- \& S-methadone, R- \& S-EDDP in plasma and DBS were quantified using HPLC-MS/MS (API 4000, Applied Biosystems) by the Indiana University Simon Cancer Center Clinical Pharmacology Analytical Core. Plasma samples and standards were prepared by adding diphenhydramine (internal standard, $20 \mathrm{ng}$ ) and $0.1 \mathrm{M}$ phosphate buffer (200 $\mu \mathrm{L}, \mathrm{pH} 7.4)$ to $200 \mu \mathrm{L}$ plasma. For DBS samples, a single punch (6 mm) was placed in a micro-centrifuge tube and $10 \mu \mathrm{L}$ of $1 \mathrm{ng} / \mu \mathrm{L}$ diphenhydramine and water were added to the sample. Plasma and DBS samples were extracted with ethyl acetate, the supernatant transferred to a clean tubes, evaporated to dryness, and reconstituted with $50 \mu \mathrm{L}$ of $10 \%$ acetonitrile in $0.1 \%$ formic acid $(\mathrm{pH} 6.5)$. Samples (10 $\mu \mathrm{L}$ ) were injected onto an Agilent $1200 \mathrm{HPLC}$ equipped with a Leap CTC Autosampler, separated on a Chiral-AGP 150X4.6mm $5 \mu$ column (Chiral Technologies, Daicel Group, West Chester, PA) over 15 minutes with acetonitrile (gradient from $10-34 \%$ ) in $0.1 \%$ formic acid ( $\mathrm{pH}$ 6.5). R and S Methadone, $\mathrm{m} / \mathrm{z}$ 310.3/265.0; $\mathrm{R}$ and S EDDP, $\mathrm{m} / \mathrm{z}$ 278.3/234.0; and diphenhydramine, $\mathrm{m} / \mathrm{z}$ 256.2/167.0 were quantified on an ABSciex $4000 \mathrm{MS} / \mathrm{MS}$. Based on a previous report, it was assumed the R-entantiomer EDDP elutes prior to the S-enantiomer. ${ }^{26}$ The lower limit of quantification for R- and Smethadone was $0.05 \mathrm{ng} / \mathrm{mL}$ for plasma and $1 \mathrm{ng} / \mathrm{mL}$ for DBS, and for R- and S- EDDP was $0.025 \mathrm{ng} / \mathrm{mL}$ for plasma and $0.5 \mathrm{ng} / \mathrm{mL}$ for DBS.

\section{Statistical analysis}


Maternal and infant methadone and EDDP concentrations were determined in plasma and cord blood at delivery. Maternal concentration data were obtained at 2 timepoints. The elimination rate constant $\left(k_{e}\right)$ was determined by linear extrapolation of log transformed concentration vs. time data. Maternal plasma concentrations were linearly extrapolated from 0 to 24 hours to estimate maternal area under the concentration time curve profile $\left(\mathrm{AUC}_{24 \mathrm{~h}}\right)$. Infant ke was calculated by linear extrapolation of the log-transformed DBS concentrations obtained from cord blood and at time of the newborn screen vs. time data. AUC inf was estimated as the cord concentration $/ \mathrm{k}_{\mathrm{e}}$. Maternal oral clearance was estimated as methadone dose/AUC(0-24h) and half-life as $\ln (2) / k_{e}$. Due to timing of maternal blood draws and variability in plasma concentrations, maternal ke could only be estimated for 7 of the 20 women in the study.

Associations between methadone dose, DBS or plasma concentrations of methadone and EDDP metabolites, total \& peak neonatal morphine dose and neonatal length of stay (LOS) were evaluated by linear regression analysis ( $R$ 3.3.1). ${ }^{27}$ This study was designed as a pilot to assess feasibility for a larger clinical study, so no formal power analysis was conducted.

\section{RESULTS}

Twenty-six women were enrolled in the study and 20 women (23-42 years) and neonatal (EGA $\left.36^{6}-41^{2}\right)$ dyads completed the study. Six women did not complete the study due to inability to collect blood samples $(n=5)$ or noncompliance with methadone therapy $(n=1)$. Data from these six women were excluded from all analyses. The median maternal methadone dose at delivery was $112 \mathrm{mg} / \mathrm{d}$ (range 60-180 mg/d). Sixteen neonates (80\%) experienced NAS requiring morphine treatment, and $3(19 \%)$ 
required adjunct therapy with phenobarbital. Demographic characteristics did not differ between those who did and did not develop NAS (Table 1). While the urine drug screens were negative for other opioids in all women, the meconium drug screen was positive for substances other than methadone in 6 infants ( 1 for amphetamines, 1 for cannabinoids, 1 for cocaine, 1 for barbiturates and opioids, 2 for other opioids). Five of these infants developed NAS ( $p>0.05)$. As expected, infants with NAS had significantly longer hospitalization than those without NAS ( 29 vs. 6 days, $p=0.0029$ ).

\section{DBS vs. Plasma Concentrations}

The DBS:plasma ratios from cord blood were significantly higher than maternal ratios ( $p \leq 0.05$, Figure 1). Therefore, maternal and cord blood DBS:plasma correlations were compared separately. In both maternal and cord blood samples Rand S-methadone were well-correlated $\left(R\right.$-methadone maternal $R^{2}=0.70, p<0.001$ and cord blood $R^{2}=0.87, p<0.001$; S-Methadone maternal $R^{2}=0.76, p<0.001$ and cord blood $\left.\mathrm{R}^{2}=0.76, \mathrm{p}<0.001\right)$. However, $\mathrm{R}$ - and S-EDDP DBS and plasma concentrations were only weakly correlated (R-EDDP maternal $R^{2}=0.41, p<0.001$ and cord blood $R^{2}=0.30$, $p=0.03$; S-EDDP maternal $R^{2}=0.45, p<0.001$ and cord blood $R^{2}=0.12, p=0.13$ ).

\section{Methadone Concentration and Dose}

Maternal R- and S-methadone plasma concentrations at delivery were weakly correlated with maternal methadone dose $\left(R^{2}=0.55, p=0.0006\right.$ and $R^{2}=0.42, p=$ 0.004 , respectively, Figure $2 A-B)$. In contrast, maternal concentrations of $R$ and SEDDP metabolites were not correlated with maternal methadone dose. Correlations between maternal dose and cord blood concentrations of parent drug and metabolite were significant, but weak $\left(R^{2} \leq 0.35\right.$, Figure $\left.2 C-D\right)$. 


\section{Neonatal Abstinence Syndrome}

Maternal R-methadone concentrations were $146 \pm 74 \mathrm{ng} / \mathrm{mL}$ vs. $202 \pm 65$ $\mathrm{ng} / \mathrm{mL}$ and cord blood concentrations at delivery were $103 \pm 63 \mathrm{ng} / \mathrm{mL}$ and $150 \pm 71$ $\mathrm{ng} / \mathrm{mL}$ in infants who did and did not experience NAS, respectively $(p=0.20)$. This trend towards neonates who experienced NAS having statistically non-significant lower maternal delivery and cord blood concentrations of methadone and EDDP metabolites than those who did not experience NAS was also observed with S-methadone, REDDP, and S-EDDP (Table S1). NAS was associated with faster maternal oral clearance of R-methadone, R-EDDP, and S-EDDP $(p=0.0086,0.048,0.035$, respectively).

Neonatal withdrawal was assessed by Finnegan scores. Length of stay was positively correlated with maximum Finnegan score $\left(p=0.003 ; R^{2}=0.36\right)$. Although Finnegan score was not correlated with maternal concentrations at delivery or AUCs of R- or S-methadone or R- or S-EDDP, lower maternal AUC of R-methadone and SEDDP were associated with longer length of hospital stay $\left(p=0.0029 ; R^{2}=0.74\right.$; and $p$ $=0.0409 ; R^{2}=0.47$, respectively). There was no association between neonatal AUC of methadone or EDDP with Finnegan scores or length of stay.

Among the 16 infants experiencing NAS, the peak dose of morphine per kg body weight required by neonates was associated with maternal concentration of R- and Smethadone, R-EDDP, and S-EDDP ( $p<0.02, R^{2}>0.38$, Figure 3$)$. Infants born to mothers with higher plasma concentrations had increased peak morphine doses. Similarly, higher R-methadone, R-EDDP, and S-EDDP cord blood concentrations were associated with higher maximum morphine doses/kg body weight $\left(p<0.01, R^{2}>0.33\right)$. 
While the trend was similar for S-methadone, the association with increased peak morphine doses was not significant $\left(p=0.15, R^{2}=0.08\right)$. Total morphine doses were not associated with maternal or cord blood concentrations of drug or metabolites at delivery.

Cord DBS to maternal plasma concentration ratios of drug and metabolites were highly variable. $\mathrm{R}$ - and S- methadone and S-EDDP had median cord DBS to maternal plasma ratios of 0.77 (range $0.47-1.21), 0.65(0.004-1.15)$, and $0.78(0.26-2.5)$, respectively. R-EDDP was more likely to have higher fetal exposure, with cord to maternal plasma ratios of $1.23(0.49-3.36)$. There was no relationship between risk of NAS and cord to maternal ratios. Although trends were observed, there were no significant associations between half-life or AUC and severity of NAS as assessed by Finnegan score, total morphine dose, or peak morphine dose. Similarly, no significant correlations were observed for neonatal half-life and $A U C_{\infty}(N=17)$ of methadone or EDDP enantiomers and severity of NAS.

\section{DISCUSSION}

This study assessed the plasma and DBS concentrations of R- and S-methadone and their primary metabolite R- and S-EDDP. Consistent with other studies, ${ }^{12,20}$ we found a wide inter-individual variability in methadone concentrations in both pregnant women and their infants. It is widely recognized that a number of physiologic changes occur during pregnancy which alter drug disposition. ${ }^{15-17}$ During pregnancy, there is a marked increase in methadone oral clearance, and many patients experience the need for a dose increase or split dosing of methadone in order to quell increased withdrawal symptoms. ${ }^{17,28,29}$ This may be due in part to the fact that both CYP2B6 and CYP19 
(aromatase) are induced by estradiol and progesterone. ${ }^{30-32}$ In addition, CYP19, which is highly expressed in the placenta, has been shown to metabolize methadone..$^{33,34}$

Cord blood concentrations of methadone were $65-77 \%$ of maternal plasma concentrations. A prior study of 15 mother-infant pairs measured maternal plasma and cord blood plasma concentrations of R-methadone using HPLC-MS and found a median cord blood:maternal ratio of 0.41 (range $0.19-0.56){ }^{20}$ The differences in cord blood and plasma concentrations between studies may be related to our use of dried blood spot sampling. When comparing dried blood spot concentrations at delivery, the cord to maternal ratio for R-methadone was 0.6 and for S-methadone it was 0.4 . Nanosvkaya et al. demonstrated that placental microsomes can metabolize methadone, although to a much lower extent than human liver microsomes ${ }^{33}$. Thus, the higher R-EDDP and SEDDP cord to maternal ratios observed are likely due to placental metabolism of methadone.

Some studies have demonstrated that larger maternal methadone dosages in late pregnancy were associated with greater neonatal concentrations and increased risk of withdrawal, ${ }^{18,19,22,35-40}$ but others refuted a correlation. ${ }^{11,23,41-44}$ Cumulative fetal exposure can be expected to vary among infants born to mothers on equivalent methadone regimens. Methadone concentrations in cord blood and at 48 hours of age ${ }^{23}$ as well as the rate of decline in neonatal serum concentration, ${ }^{22}$ appear to correlate with NAS signs. Kuschel et al. ${ }^{23}$ found that infants who required rescue treatment had lower cord blood methadone concentrations and that, in all but one infant, methadone concentrations were undetectable in the serum at 48 hours. Doberczak noted that faster declines in postnatal blood methadone concentrations were associated 
with more severe CNS withdrawal. ${ }^{22}$ They also found a positive correlation between the rate of decline in neonatal plasma concentration between day 1 and days 3-4 after delivery and severity of CNS signs of withdrawal, as measured by the Lipsitz scoring system. We found that infants with greater exposure to R-methadone as measured by cord blood concentration had more severe NAS. However, we were unable to detect a correlation between methadone half-life and NAS severity.

Despite the long-standing use of methadone and experiences with NAS, there is virtually no data available on methadone pharmacokinetics in infants. A population pharmacokinetic study by Ward et al used pooled data from 4 studies of methadone metabolism in neonates and children and found that methadone clearance in neonates was similar to that in older children and adults. ${ }^{45}$ The activity of most drug metabolizing enzymes begins to increase dramatically immediately following birth. ${ }^{46}$ As we were only able to obtain a single sample from neonates following delivery in addition to cord blood, we could only estimate half-life by linear regression of drug concentrations at birth and the time of the newborn screen. However, it is likely that there is variability in the rate of enzyme development that is not captured by this approach. Such variability may also affect the risk and severity of NAS in neonates.

This study does have several limitations, most importantly a small sample size which precludes us from discerning any definitive associations and limits our ability to perform multiple comparisons to correct for known factors associated with NAS. For instance, three women whose infants experienced NAS were also being treated with antidepressants while none of the mothers whose infants were not diagnosed with NAS took antidepressants. We also included late preterm infants, which may have 
introduced a confounding factor in terms of severity of NAS. It is known that preterm infants have overall less severe NAS compared to term infants. Doberczak et al. studied NAS in 178 term and 34 preterm infants and found that $81 \%$ of the term infants required pharmacotherapy for NAS, versus $59 \%$ of the preterm infants. ${ }^{38}$ We only had three infants born less than 37 weeks gestation (one at 35.1 and two at 36.9 weeks), all of whom experienced NAS. The trends we observed in this pilot need to be further explored in larger studies. Neonatal sample collection was limited to cord blood and DBS samples at the time of neonatal screening. DBS collection appears to be an alternative to plasma collection for $\mathrm{R}$ - and $\mathrm{S}$-methadone, as $\mathrm{R}^{2}$ value is $>0.74$. However, there was only weak association between DBS and plasma concentrations for the EDDP metabolites $\left(R^{2}=0.4\right)$. This may be due to a number of factors including collection technique and interindividual differences in hematocrit. 47,48 Unfortunately, hematocrits were not available from patients in this study. Thus, it is difficult to assess the relationship between neonatal EDDP concentrations and outcomes. We used Whatman-903 DBS cards; other DBS collection matrices may be more suitable for EDDP. Only two plasma or DBS samples were available from each woman. Time between methadone dosing and sample collection also varied between patients, which impacts interpretation of cord:maternal ratio. In women who labored more than 24 hours, methadone was often re-dosed between study samples, leading to difficulties in estimating terminal elimination rate. While the diagnosis of NAS and morphine dosing was set by standardized clinical protocols based on the Finnegan score, we recognize that this is a subjective assessment scale. 
In spite of these limitations, this study demonstrates the feasibility of recruiting pregnant women with opioid abuse disorder and their neonates into a prospective pharmacokinetic-pharmacodynamic study. We also utilized a sensitive mass spectroscopy technique that allowed us to detect individual enantiomers of methadone and EDDP. Further studies assessing the relationship between in utero or postnatal exposure to methadone enantiomers may inform models to predict which infants will experience NAS, and the severity of NAS. These models could then enable earlier and more aggressive treatment of NAS, reducing length of neonatal stay and morbidities associated with severe withdrawal.

\section{CONCLUSSIONS}

This study demonstrates that DBS is a suitable alternative for methadone quantification, especially when sample volume is limited such as in neonates. However, the DBS method employed here is not sufficient for measuring EDDP metabolites. This pilot data indicates that maternal methadone AUC and cord blood concentration at delivery may be potential markers for NAS severity as measured by Finnegan score. However, EDDP metabolite concentrations do not appear to be correlated with NAS. Additional studies, with more objective measures of NAS, are needed to further understand the association between maternal and infant exposure to methadone and NAS.

\section{Acknowledgments}

This study was supported by K23 HD071134-01A1 (NICHD) and UL1TR001108 Indiana Clinical and Translational Sciences Institute (Pediatric Project Development 
Team Funding). Analytical work was performed by the Clinical Pharmacology Analytical Core laboratory, a core laboratory of the Indiana University Melvin and Bren Simon Cancer Center supported by the National Cancer Institute grant P30 CA082709. 


\section{REFERENCES}

1. Patrick SW, Davis MM, Lehmann CU, Cooper WO. Increasing incidence and geographic distribution of neonatal abstinence syndrome: United States 2009 to 2012. Journal of perinatology : official journal of the California Perinatal Association 2015;35(8):650-655

2. U.S. Department of Health and Human Services. HHS Acting Secretary Declares Public Health Emergency to Address National Opioid Crisis. In: HHS Press Office; 2017

3. Patrick SW, Schumacher RE, Benneyworth BD, et al. Neonatal abstinence syndrome and associated health care expenditures: United states, 2000-2009. JAMA : the journal of the American Medical Association 2012;307(18):1934-1940

4. Hudak ML, Tan RC. Neonatal drug withdrawal. Pediatrics 2012;129(2):e540-560

5. Tolia VN, Patrick SW, Bennett MM, et al. Increasing incidence of the neonatal abstinence syndrome in U.S. neonatal ICUs. The New England journal of medicine 2015;372(22):2118-2126

6. ACOG Committee Opinion No. 524: Opioid abuse, dependence, and addiction in pregnancy. Obstet Gynecol 2012;119(5):1070-1076

7. Substance Abuse and Mental Health Services Administration. National Survey of Substance Abuse Treatment Services (N-SSATS): 2018. Data on Substance Abuse Treatment Facilities. In. Rockville, MD Substance Abuse and Mental Health Services Administration,; 2019

8. Mattick RP, Breen C, Kimber J, Davoli M. Methadone maintenance therapy versus no opioid replacement therapy for opioid dependence. Cochrane Database Syst Rev 2009(3):Cd002209

9. Kristensen K, Christensen CB, Christrup LL. The mu1, mu2, delta, kappa opioid receptor binding profiles of methadone stereoisomers and morphine. Life Sci 1995;56(2):PI45-50

10. Begre $S$, von Bardeleben $U$, Ladewig $D$, et al. Paroxetine increases steady-state concentrations of (R)-methadone in CYP2D6 extensive but not poor metabolizers. Journal of clinical psychopharmacology 2002;22(2):211-215

11. Cleary BJ, Donnelly J, Strawbridge J, et al. Methadone dose and neonatal abstinence syndromesystematic review and meta-analysis. Addiction (Abingdon, England) 2010;105(12):2071-2084

12. Eap CB, Bourquin M, Martin J, et al. Plasma concentrations of the enantiomers of methadone and therapeutic response in methadone maintenance treatment. Drug and alcohol dependence 2000;61(1):47-54

13. Kharasch ED, Regina KJ, Blood J, Friedel C. Methadone Pharmacogenetics: CYP2B6 Polymorphisms Determine Plasma Concentrations, Clearance, and Metabolism. Anesthesiology 2015;123(5):1142-1153

14. Kharasch ED. Current Concepts in Methadone Metabolism and Transport. Clinical pharmacology in drug development 2017;6(2):125-134

15. Abduljalil K, Furness P, Johnson TN, Rostami-Hodjegan A, Soltani H. Anatomical, physiological and metabolic changes with gestational age during normal pregnancy. Clinical pharmacokinetics 2012;51(6):365-396

16. Dallmann A, Ince I, Meyer M, et al. Gestation-Specific Changes in the Anatomy and Physiology of Healthy Pregnant Women: An Extended Repository of Model Parameters for Physiologically Based Pharmacokinetic Modeling in Pregnancy. Clin Pharmacokinet 2017

17. Wolff K, Boys A, Rostami-Hodjegan A, Hay A, Raistrick D. Changes to methadone clearance during pregnancy. Eur J Clin Pharmacol 2005;61(10):763-768

18. Rosen TS, Pippenger CE. Disposition of methadone and its relationship to severity of withdrawal in the newborn. Addictive diseases 1975;2(1-2):169-178

19. Harper RG, Solish G, Feingold E, Gersten-Woolf NB, Sokal MM. Maternal ingested methadone, body fluid methadone, and the neonatal withdrawal syndrome. Am J Obstet Gynecol 1977;129(4):417-424 
20. Gordon AL, Lopatko OV, Somogyi AA, Foster DJ, White JM. (R)- and (S)-methadone and buprenorphine concentration ratios in maternal and umbilical cord plasma following chronic maintenance dosing in pregnancy. Br J Clin Pharmacol 2010;70(6):895-902

21. Nanovskaya TN, Nekhayeva IA, Hankins GD, Ahmed MS. Transfer of methadone across the dually perfused preterm human placental lobule. Am J Obstet Gynecol 2008;198(1):126.e121-124

22. Doberczak TM, Kandall SR, Friedmann P. Relationship between maternal methadone dosage, maternal-neonatal methadone levels, and neonatal withdrawal. Obstet Gynecol 1993;81(6):936940

23. Kuschel CA, Austerberry L, Cornwell M, Couch R, Rowley RS. Can methadone concentrations predict the severity of withdrawal in infants at risk of neonatal abstinence syndrome? Archives of disease in childhood Fetal and neonatal edition 2004;89(5):F390-393

24. Dyer KR, Foster DJ, White JM, et al. Steady-state pharmacokinetics and pharmacodynamics in methadone maintenance patients: comparison of those who do and do not experience withdrawal and concentration-effect relationships. Clin Pharmacol Ther 1999;65(6):685-694

25. Finnegan LP, Kron RE, Connaughton JF, Emich JP. Assessment and treatment of abstinence in the infant of the drug-dependent mother. International journal of clinical pharmacology and biopharmacy 1975;12(1-2):19-32

26. Johansen SS, Linnet K. Chiral analysis of methadone and its main metabolite EDDP in postmortem blood by liquid chromatography-mass spectrometry. Journal of analytical toxicology 2008;32(7):499-504

27. R Development Core Team. R: A Language and Environment for Statistical Computing. In. Vienna, Austria 2008

28. Pond SM, Kreek MJ, Tong TG, Raghunath J, Benowitz NL. Altered methadone pharmacokinetics in methadone-maintained pregnant women. J Pharmacol Exp Ther 1985;233(1):1-6

29. Bogen DL, Perel JM, Helsel JC, et al. Pharmacologic evidence to support clinical decision making for peripartum methadone treatment. Psychopharmacology 2013;225(2):441-451

30. Koh KH, Jurkovic S, Yang K, et al. Estradiol induces cytochrome P450 2B6 expression at high concentrations: implication in estrogen-mediated gene regulation in pregnancy. Biochemical pharmacology 2012;84(1):93-103

31. Dickmann L, Isoherranen N. Quantitative prediction of CYP2B6 induction by estradiol during pregnancy: potential explanation for increased methadone clearance during pregnancy. Drug Metab Dispos 2013;41(2):270-274

32. Choi SY, Koh KH, Jeong H. Isoform-specific regulation of cytochromes $\mathrm{P} 450$ expression by estradiol and progesterone. Drug Metab Dispos 2013;41(2):263-269

33. Nanovskaya TN, Deshmukh SV, Nekhayeva IA, et al. Methadone metabolism by human placenta. Biochemical pharmacology 2004;68(3):583-591

34. Hieronymus TL, Nanovskaya TN, Deshmukh SV, et al. Methadone metabolism by early gestational age placentas. Am J Perinatol 2006;23(5):287-294

35. Madden JD, Chappel JN, Zuspan F, et al. Observation and treatment of neonatal narcotic withdrawal. Am J Obstet Gynecol 1977;127(2):199-201

36. Ostrea EM, Chavez CJ, Strauss ME. A study of factors that influence the severity of neonatal narcotic withdrawal. The Journal of pediatrics 1976;88(4 Pt 1):642-645

37. Strauss ME, Andresko M, Stryker JC, Wardell JN. Relationship of neonatal withdrawal to maternal methadone dose. The American journal of drug and alcohol abuse 1976;3(2):339-345

38. Doberczak TM, Kandall SR, Wilets I. Neonatal opiate abstinence syndrome in term and preterm infants. The Journal of pediatrics 1991;118(6):933-937

39. Dashe JS, Sheffield JS, Olscher DA, et al. Relationship between maternal methadone dosage and neonatal withdrawal. Obstet Gynecol 2002;100(6):1244-1249 
40. Liu AJ, Jones MP, Murray H, Cook CM, Nanan R. Perinatal risk factors for the neonatal abstinence syndrome in infants born to women on methadone maintenance therapy. The Australian \& New Zealand journal of obstetrics \& gynaecology 2010;50(3):253-258

41. Brown HL, Britton KA, Mahaffey D, et al. Methadone maintenance in pregnancy: a reappraisal. Am J Obstet Gynecol 1998;179(2):459-463

42. Mack G, Thomas D, Giles W, Buchanan N. Methadone levels and neonatal withdrawal. Journal of paediatrics and child health 1991;27(2):96-100

43. Berghella V, Lim PJ, Hill MK, et al. Maternal methadone dose and neonatal withdrawal. Am J Obstet Gynecol 2003;189(2):312-317

44. Seligman NS, Almario CV, Hayes EJ, et al. Relationship between maternal methadone dose at delivery and neonatal abstinence syndrome. The Journal of pediatrics 2010;157(3):428-433, 433.e421

45. Ward RM, Drover DR, Hammer GB, et al. The pharmacokinetics of methadone and its metabolites in neonates, infants, and children. Paediatric anaesthesia 2014;24(6):591-601

46. Blake MJ, Castro L, Leeder JS, Kearns GL. Ontogeny of drug metabolizing enzymes in the neonate. Seminars in fetal \& neonatal medicine 2005;10(2):123-138

47. Rowland $\mathrm{M}$, Emmons GT. Use of dried blood spots in drug development: pharmacokinetic considerations. The AAPS journal 2010;12(3):290-293

48. O'Mara M, Hudson-Curtis B, Olson $\mathrm{K}$, et al. The effect of hematocrit and punch location on assay bias during quantitative bioanalysis of dried blood spot samples. Bioanalysis 2011;3(20):23352347 
Table 1. Maternal demographics and neonatal outcomes in neonates with vs. without NAS diagnoses.

\begin{tabular}{|c|c|c|c|}
\hline Characteristic & $\begin{array}{l}\text { NAS } \\
(\mathrm{N}=16)\end{array}$ & $\begin{array}{l}\text { No NAS } \\
(\mathrm{N}=4)\end{array}$ & $P$-value \\
\hline Methadone dose (mg) & $110(60-170)$ & $131(95-180)$ & 0.20 \\
\hline Maternal age (years) & $28(23-42)$ & $35(24-35)$ & 0.25 \\
\hline Race & & & 0.18 \\
\hline Black & $0(0 \%)$ & $2(50 \%)$ & \\
\hline White & $15(94 \%)$ & $2(50 \%)$ & \\
\hline Other & $1(6 \%)$ & $0(0 \%)$ & \\
\hline Ethnicity & & & 1.0 \\
\hline Hispanic & $1(6 \%)$ & $0(0 \%)$ & \\
\hline Non-Hispanic & $15(94 \%)$ & $4(100 \%)$ & \\
\hline Height $(\mathrm{cm})$ & $164(152-175)$ & $160(152-173)$ & 0.48 \\
\hline Weight $(\mathrm{kg})$ & $86(56-114)$ & $79(58-90)$ & 0.51 \\
\hline $\operatorname{BMI}\left(\mathrm{kg} / \mathrm{m}^{2}\right)$ & $31(22-43)$ & $29(23-35)$ & 0.60 \\
\hline Tobacco Smoker & $15(94 \%)$ & $3(75 \%)$ & 0.74 \\
\hline Antidepressant Use & $3(19 \%)$ & $0(0 \%)$ & 0.88 \\
\hline $\begin{array}{l}\text { Gestational age at delivery } \\
\text { (weeks) }\end{array}$ & $38(35-41)$ & $39(37-40)$ & 0.81 \\
\hline Birth Weight (kg) & $3(2.3-3.7)$ & $2.6(2.4-2.9)$ & 0.20 \\
\hline Male sex & $8(50 \%)$ & $2(50 \%)$ & 1.0 \\
\hline Breastfeeding (Yes) & $6(38 \%)$ & $3(75 \%)$ & 0.28 \\
\hline
\end{tabular}




\begin{tabular}{|l|c|c|c|}
\hline Meconium positive & $5(31 \%)$ & $1(25 \%)$ & 0.70 \\
\hline 1 minute Apgar score & $9(1-9)$ & $9(7-9)$ & 0.32 \\
\hline Peak Finnegan Score & $13(10-20)$ & $9(7-10)$ & $0.003^{*}$ \\
\hline Number of days in hospital & $29(19-55)$ & $6(5-6)$ & $0.003^{*}$ \\
\hline Peak morphine dose & $0.05(0.02-$ & & \\
(mg/day/kg) & $0.61)$ & & \\
\hline Total cumulative morphine & $5.64(1.02-$ & & \\
\hline dose (mg/kg) & $17.53)$ & & \\
\hline
\end{tabular}

* $p<0.05$ for Mann-Whitney test comparing "NAS" versus "no NAS". Values are Median (Confidence Interval) or $\mathrm{N}(\%)$. Groups were compared by $\mathrm{X}^{2}$, Fisher exact or MannWhitney test as appropriate. NAS, neonatal abstinence syndrome; BMI, body mass index 
Figure 1. Correlation between plasma and DBS concentrations of methadone and EDDP. A) R-Methadone maternal (black) $R^{2}=0.70, p<0.001$ and cord blood (blue) $R^{2}=0.87, p<0.001$; B) S-Methadone maternal (black) $R^{2}=0.76, p<0.001$ and cord blood (blue) $\left.R^{2}=0.76, p<0.001, C\right) R$-EDDP maternal (black) $R^{2}=0.41, p<0.001$ and cord blood (blue) $R^{2}=0.30, p=0.03$ and D) $S$-EDDP maternal (black) $R^{2}=0.45, p<0.001$ and cord blood (blue) $R^{2}=0.12, p=0.13$. Lines indicate linear regression fit of cord blood (blue dashed) and maternal (black solid) data.

Figure 2. Concentration of methadone (blue circles) and EDDP (red open squares) at delivery compared to maternal methadone dose in maternal plasma ( $A$ and $B$ ) and infant DBS (C and D). Lines indicate linear regression of A) Maternal plasma Rmethadone $(R M)$ concentration vs. dose (solid, $R^{2}=0.55 ; p=0.0006$ ) and $R$-EDDP (RE) vs. dose (dashed, $\mathrm{R}^{2}=0.11 ; \mathrm{p}=0.13$ ); B) Maternal plasma S-methadone (SM) concentration vs. dose $\left(R^{2}=0.42 ; p=0004\right)$ S-EDDP $(S E)$ vs. dose $\left(R^{2}=0.20 ; p=0.053\right)$; C) Cord DBS R-methadone concentration vs. dose (solid, $R^{2}=0.35 ; p=0.004$ ) R-EDDP vs. dose (dashed, $R^{2}=0.31 ; p=0.007$ ); and D.) Cord DBS S-methadone concentration vs. dose $\left(R^{2}=0.32 ; p=0.005\right)$ S-EDDP $\left(R^{2}=0.32 ; p=0.006\right)$.

Figure 3. Correlations between peak dose of morphine/kg and maternal plasma (A and B) and cord DBS concentrations at delivery (C and D). Lines indicate linear regression of $A$ ) Maternal plasma R-methadone (RM) concentration vs. peak neonatal morphine dose (solid, $R^{2}=0.54 ; p=0.004$ ) and R-EDDP (RE) vs. peak neonatal morphine dose (dashed, $\left.R^{2}=0.55 ; p=0.008\right) ; B$ ) Maternal plasma S-methadone (SM) 
concentration vs. peak neonatal morphine dose $\left(R^{2}=0.38 ; p=0.019\right)$ and maternal plasma S-EDDP (SE) concentration vs. peak neonatal morphine dose $\left(R^{2}=0.48 ; p=\right.$ 0.011); C) Cord DBS R-methadone vs. peak neonatal morphine dose (solid, $R^{2}=0.33 ; p$ $=0.012$ ) and cord DBS R-EDDP vs. peak neonatal morphine dose (dashed, $R^{2}=0.39 ; p$ $=0.006$ ); and D.) Cord DBS S-methadone concentration vs. peak neonatal morphine dose (solid, $R^{2}=0.08 ; p=0.15$ ) and S-EDDP vs. peak neonatal morphine dose (dashed, $R^{2}=0.36 ; p=0.009$ ). 
Figure 1.

A.

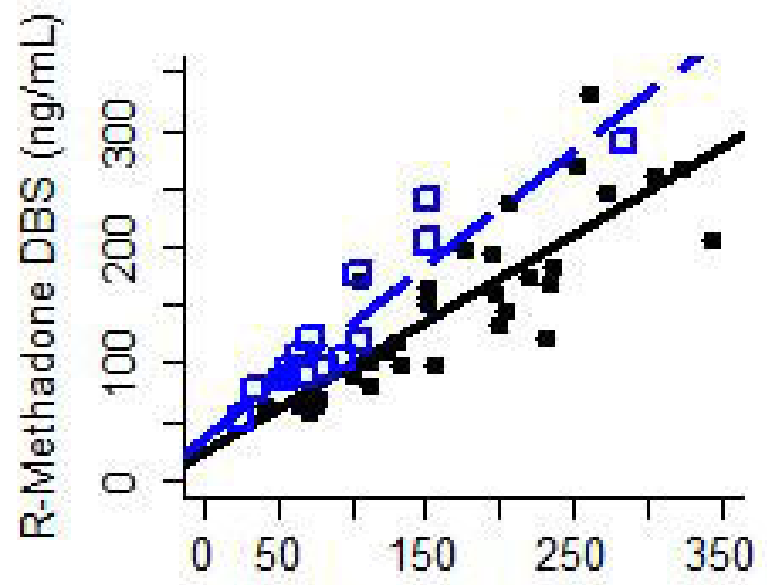

R-Methadone Plasma ( $\mathrm{ng} / \mathrm{mL}$ )

C.

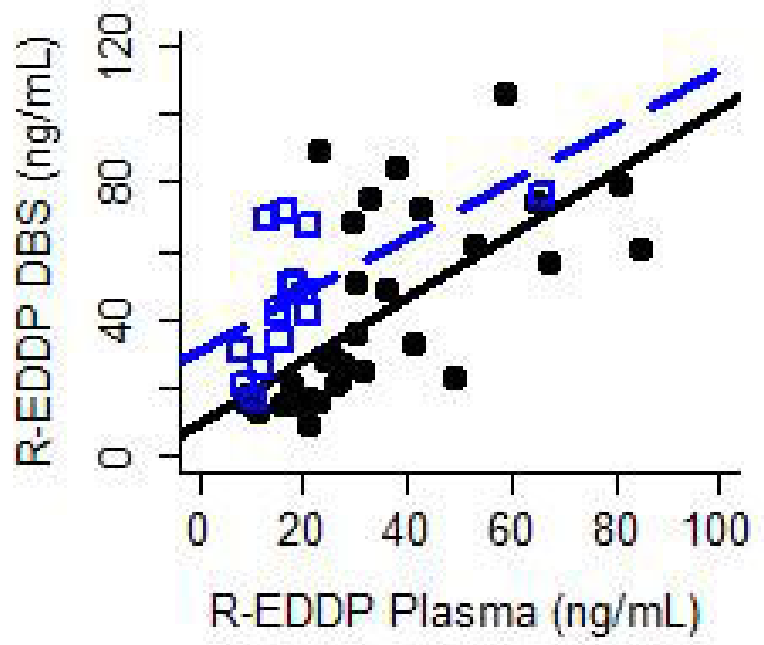

B.

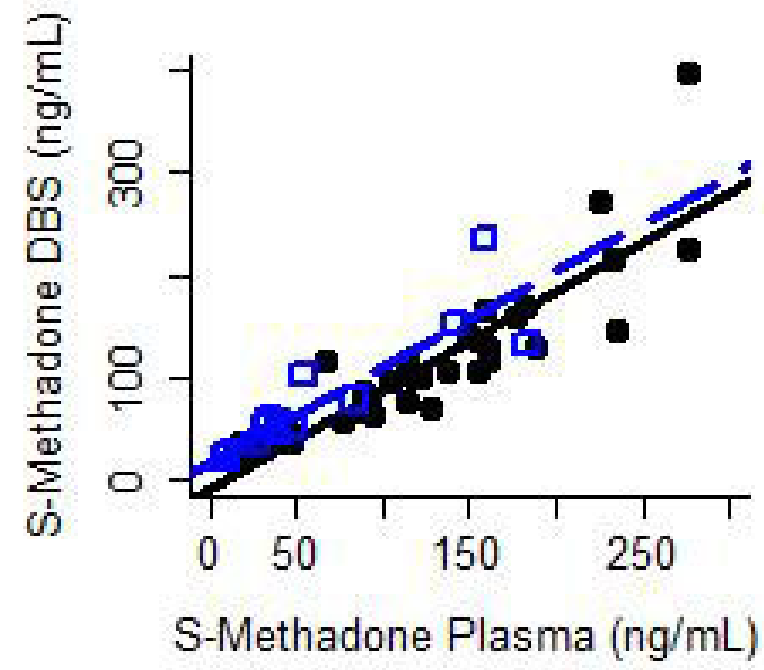

D.

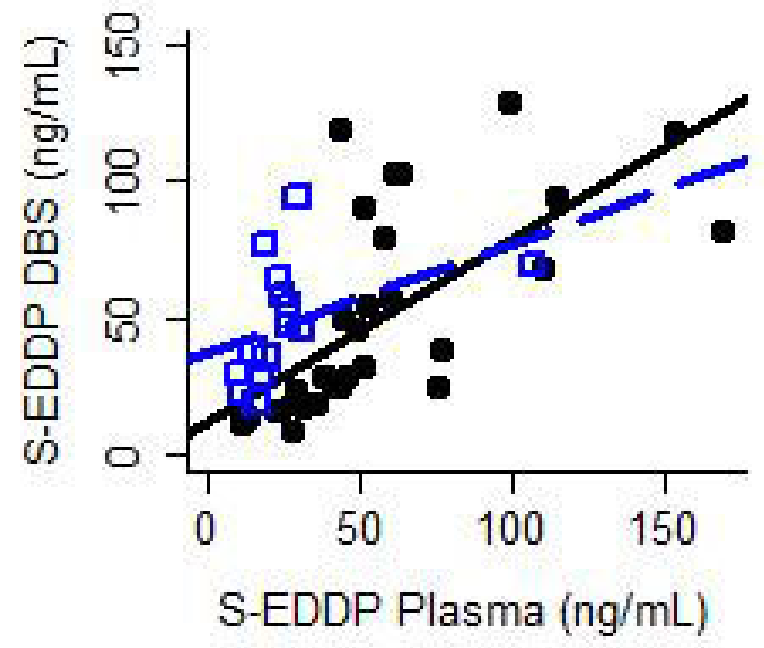


Figure 2.

A.

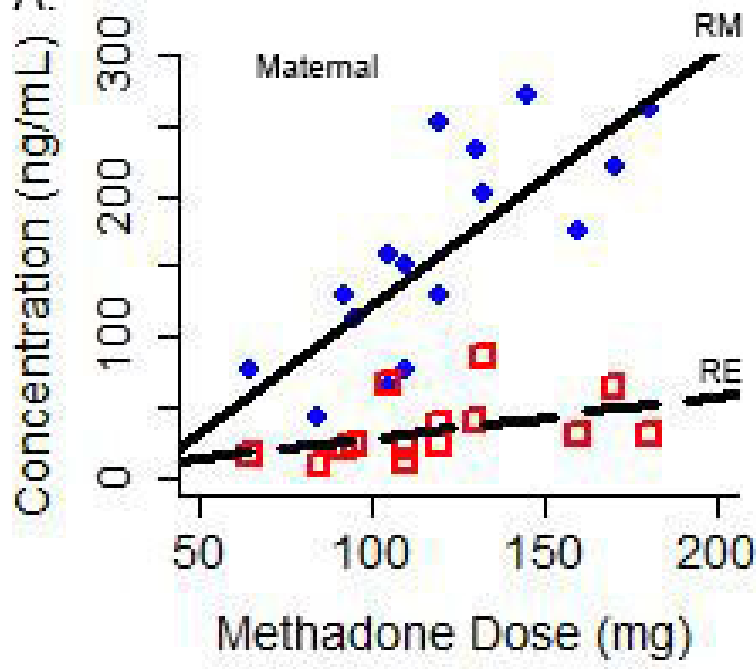

C.

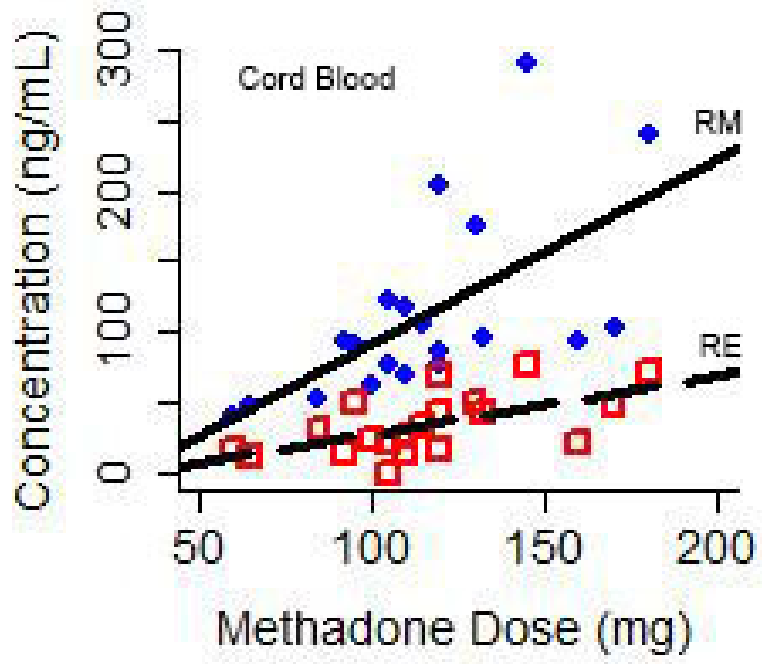

B.

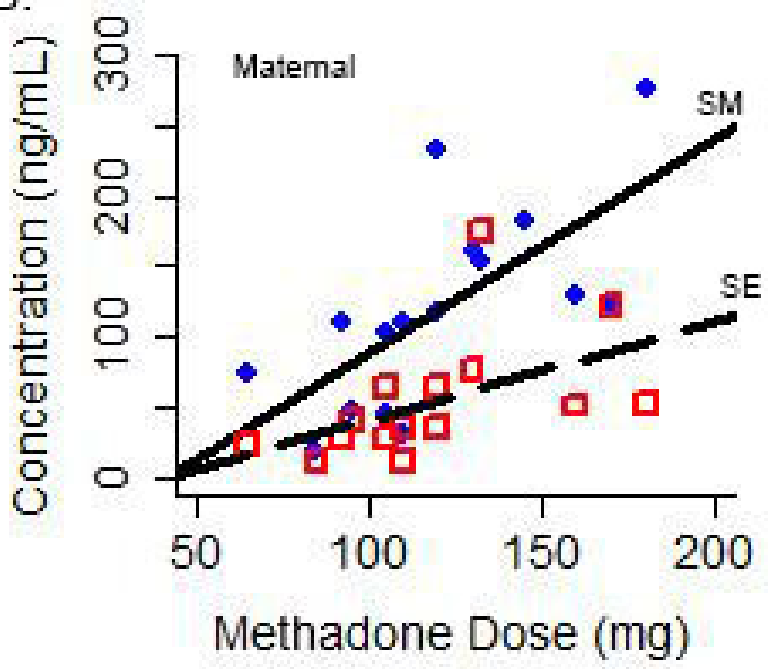

D.

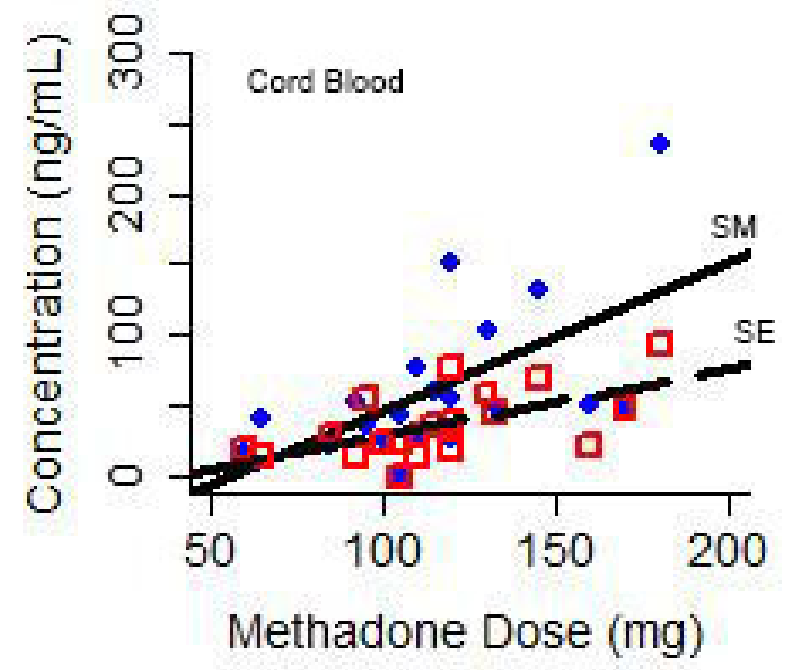


Figure 3.

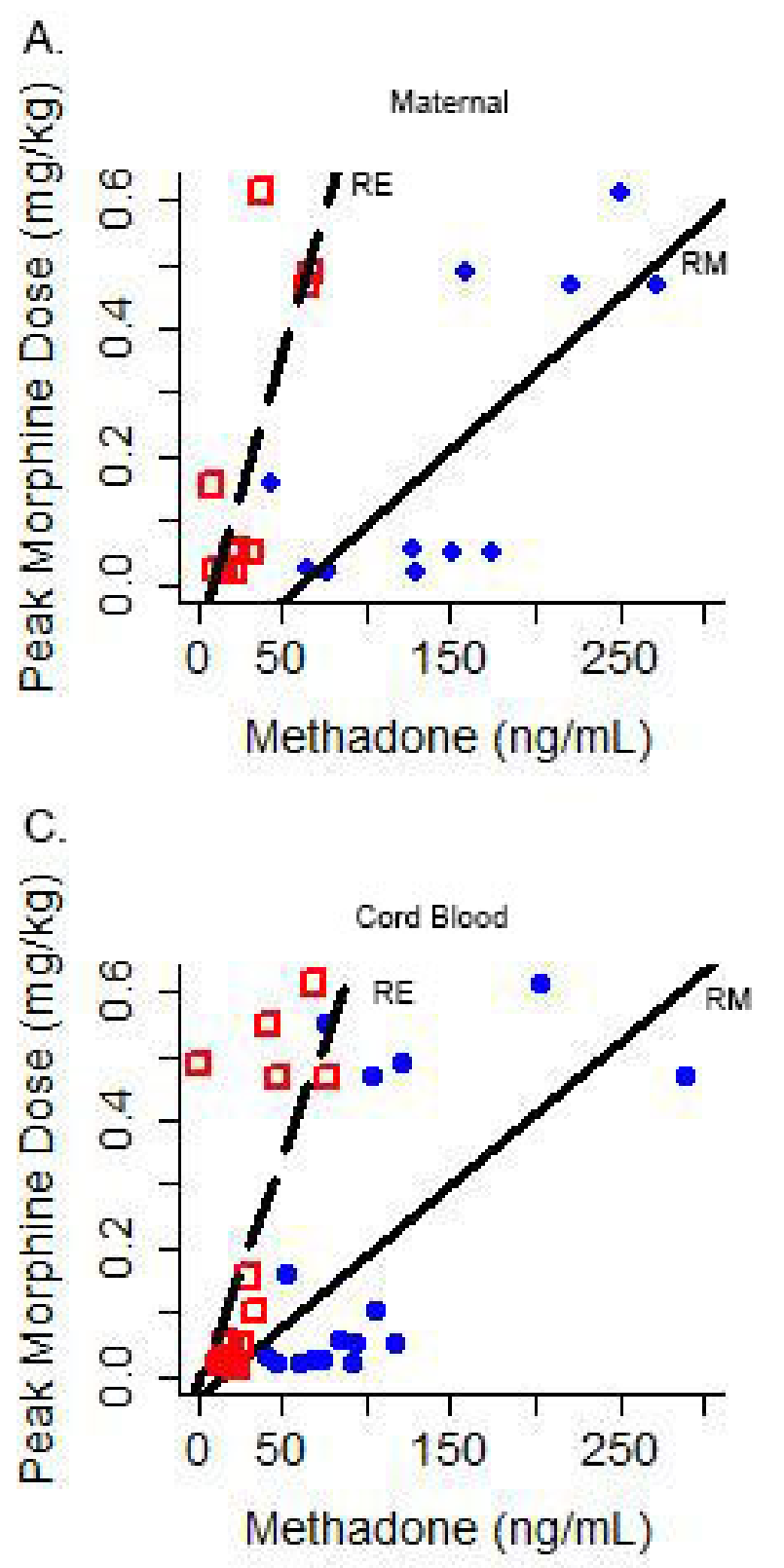

B.

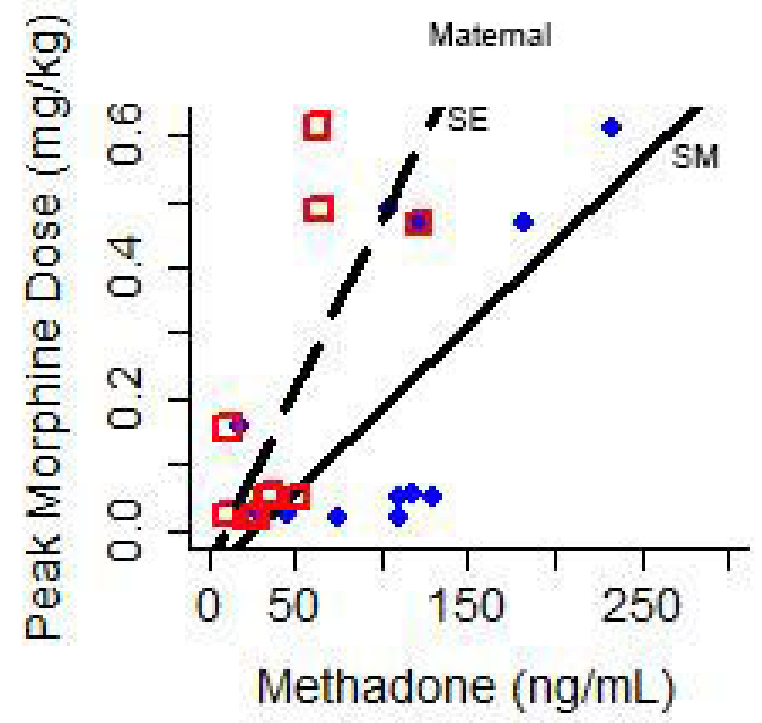

D.

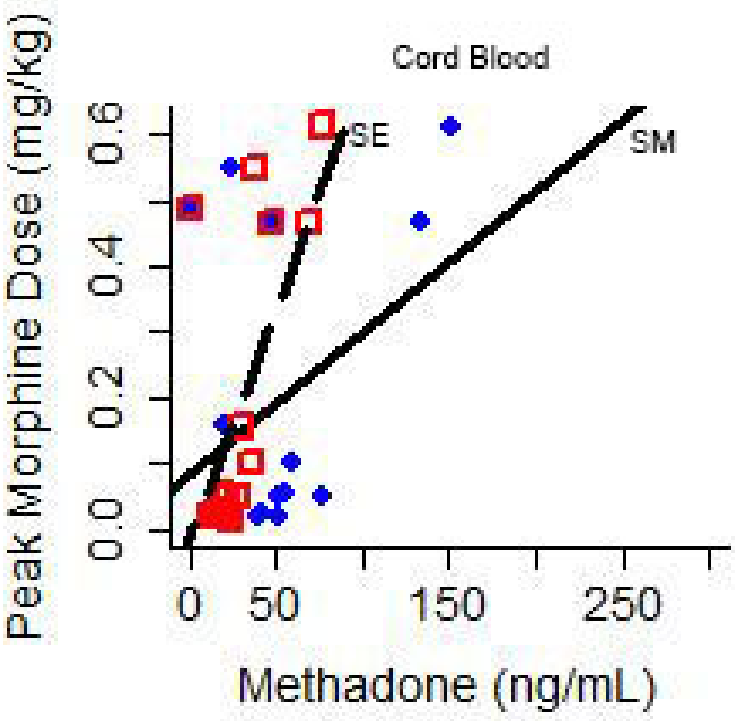


Table S1. Concentration at delivery

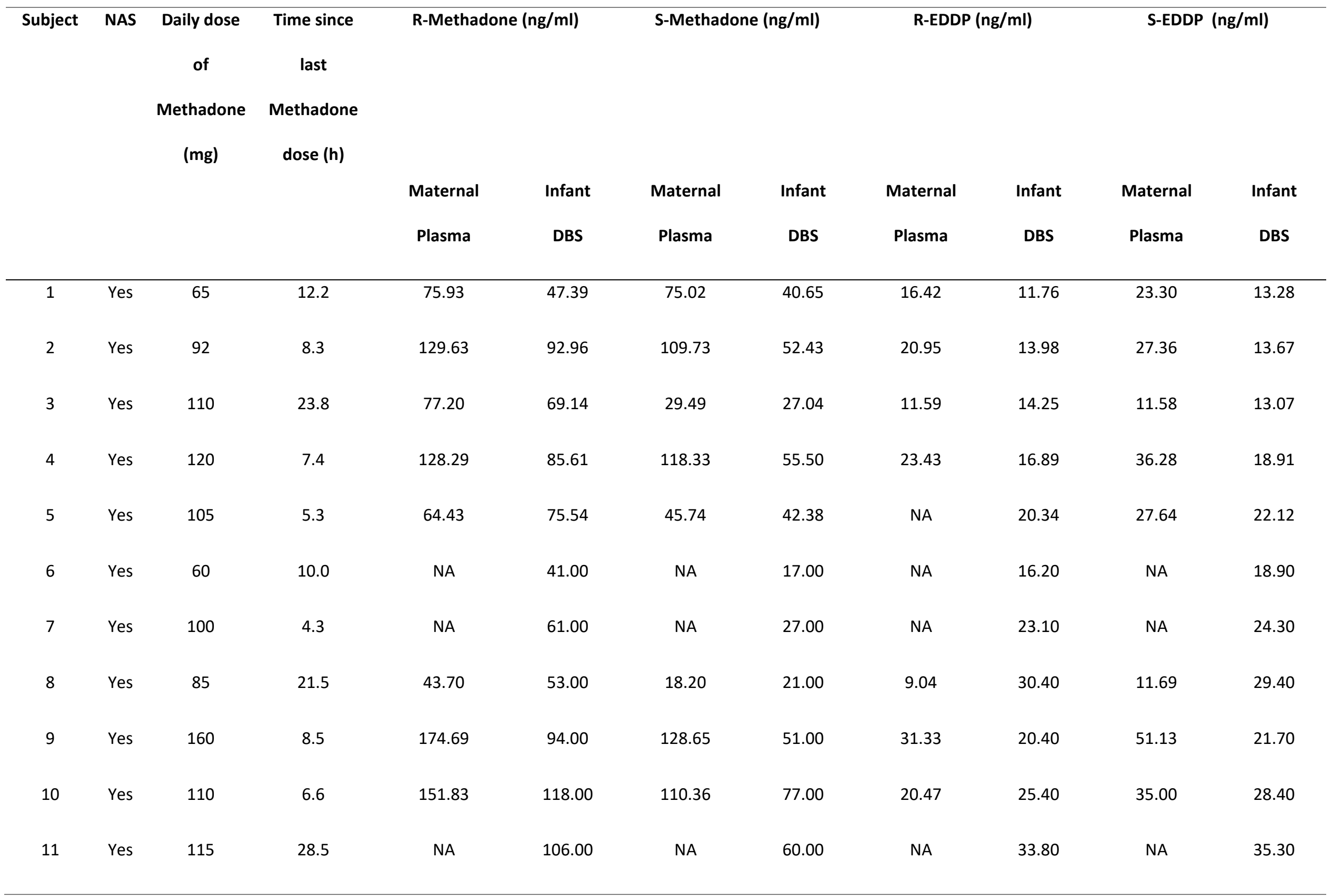




\begin{tabular}{|c|c|c|c|c|c|c|c|c|c|c|c|}
\hline 12 & Yes & 145 & 7.3 & 271.50 & 290.00 & 182.20 & 133.00 & 0.00 & 76.80 & 0.00 & 69.50 \\
\hline 13 & Yes & 170 & 1.6 & 220.50 & 104.00 & 121.70 & 47.00 & 64.90 & 47.90 & 121.89 & 47.20 \\
\hline 14 & Yes & 120 & 47.7 & NA & 77.00 & NA & 25.00 & NA & 42.20 & NA & 37.80 \\
\hline 15 & Yes & 120 & 1.5 & 251.60 & 204.00 & 233.70 & 151.00 & 37.70 & 69.30 & 63.40 & 77.30 \\
\hline 16 & Yes & 105 & 17.2 & 158.54 & 122.00 & 103.42 & 0.41 & 67.50 & NA & 64.00 & NA \\
\hline 17 & No & 132 & 1.4 & 202.60 & 96.00 & 154.50 & 51.00 & 86.13 & 41.90 & 175.50 & 45.50 \\
\hline 18 & No & 130 & 8.2 & 232.40 & 176.00 & 160.90 & 103.00 & 41.01 & 50.50 & 76.42 & 58.50 \\
\hline 19 & No & 95 & 10.4 & 111.40 & 91.00 & 48.30 & 35.00 & 24.42 & 49.20 & 41.24 & 54.60 \\
\hline 20 & No & 180 & 6.8 & 261.60 & 240.00 & 276.50 & 235.00 & 29.76 & 71.80 & 51.63 & 94.10 \\
\hline
\end{tabular}

